\title{
A genetic handle on brain circuits
}

Cre-recombinase mouse driver lines provide tools for the functional dissection of neuronal circuits.

The near future will likely bring an increase in the number of studies reporting detailed analyses of complex brain circuits and their functional involvement in mediating specific behaviors and brain functions. But to perform these types of studies, researchers need to be able to target specific cells of interest within the brain and express activity sensors or modulators in them.

Among the existing approaches for genetically targeting neuron populations is the use of Cre-driver mouse lines in which Cre recombinase is expressed under the control of gene-specific promoters. The GENSAT (Gene Expression Nervous System Atlas) project, led by Nathaniel Heintz at Rockefeller University and Charles Gerfen at the US National Institute of Mental Health, has generated over 250 Cre-driver lines using bacterial artificial chromosomes (BACs) that express the recombinase in specific neuron and glial populations of the brain. These lines were initially characterized by crossing BAC-Cre driver lines with a Rosa26-EGFP reporter line and visualizing EGFP labeling in brain sections. But this type of characterization does not always reflect the expression of the Cre recombinase in adult animals or the axonal projections and circuitry of the targeted cells.

In a recent report, Gerfen and colleagues provide additional characterization of 100 of the $250 \mathrm{BAC}$-Cre lines based on injection of adenoviral vectors (AAV) that drive Credependent expression of a fluorescent reporter. This type of labeling allows visualization of the targeted cells and their projections and is also predictive of which neurons in a given BAC-Cre line will be targeted with similar AAVs encoding Cre-dependent constructs for activity monitoring or modulation- precisely the types of experiments that the lines are likely to be used for.

Gerfen and team characterized lines with expression in neuroanatomical pathways within the cerebral cortex and basal ganglia. They found over $30 \mathrm{BAC}-\mathrm{Cre}$ lines with expression in the cortex, including lines that showed expression specific for particular cortical layers, spanning across several layers or only in subtypes of neurons within a given layer. They also characterized lines with expression in other brain structures such as the thalamus, the striatum and the thalamic nuclei. The entire data sets are available for browsing (http://GENSATcreBrains.biolucida.net), and the lines are ready for use.

\section{Erika Pastrana}

\section{RESEARCH PAPERS}

Gerfen C.R. et al. GENSAT BAC Cre-recombinase driver lines to study the functional organization of cerebral cortical and basal ganglia circuits. Neuron 80 , 1368-1383 (2013). 